\title{
Assessment and planning for oncoplastic and reconstructive breast surgery: a review and a proposed chart
}

\author{
Amit Agrawal ${ }^{1}$
}

Received: 26 March 2016 / Accepted: 24 June 2016 / Published online: 14 July 2016

(C) Springer-Verlag Berlin Heidelberg 2016. This article is published with open access at Springerlink.com

\begin{abstract}
Assessment for oncoplastic and reconstructive breast surgery (ORBS) can be time-consuming and subjective with a prolonged learning curve. Further, lack of recognition of options may lead to loss of patient choices whether available locally or not. This article reviews and incorporates essential variables into an author-designed chart with components of clinic consultation ("History" and "Examination") that classify individual cases into one of the three complexities: high, average and low. Based on these complexities, decisionmaking processes are discussed in order to achieve, safely and realistically, both subjective and objective (anticipated versus achieved) goals of ORBS. This article by summarising literature around common ORBS variables attempts to streamline decision-making process as well as charts out variables to facilitate audit of decision-making and case selection process. Level of Evidence: Not ratable
\end{abstract}

Keywords Breast - Oncoplastic · Reconstructive ·

Mammaplasty $\cdot$ Perforator $\cdot$ Implant

\section{Introduction}

Assessment and planning for oncoplastic and reconstructive breast surgery (ORBS) often necessitates multiple

Presentations Presented in the Annual Association of Breast Surgeons (UK) meeting, Bournemouth, June 2015

Amit Agrawal

amit.agrawal@addenbrookes.nhs.uk

1 Cambridge University Hospitals, Cambridge Breast Unit, Box 97, Cambridge CB22 7QT, UK consultations and collation of variables, which can be timeconsuming. In addition, application of these anatomical and tumoural variables is largely subjective with a prolonged learning curve, and inadequate assessment may deny patients all available options even if not available locally [1]. This article discusses essential variables leading to essential goals of surgery, which may contribute to minimising these limitations.

It also reviews and discusses the essential variables of the two components of clinic consultation ("History" and "Examination") for planning ORBS. These variables are incorporated into an organised and simplistic chart (Table 1) in an attempt to streamline consultation factors into risk categories that could help decision-making process leading to baseline-anticipated goals later contrasted with actual-achieved goals of oncoplastic options (Table 2).

\section{Literature review and proposed chart component discussion}

Table 1 charts the essential components of clinical assessment for ORBS that help decision-making process as well as demonstrate to the patient the risk factors. Seven essential components each in the "History" and "Examination" sections are categorised into "relative" complex categories of higher, average and lower complexity that help the author stratify risk-factors that guide planning of safe and realistic options for individual patient. These 14 individual components of "History" and "Examination" are discussed further along with summarisation of current literature.

Table 2 charts the seven common/essential components of the goals of surgery that are discussed further in the text along with literature review. The utility of this chart is in 
Table 1 Table showing common components of clinical assessment for oncoplastic and reconstructive breast surgery and categorising into "relative" complex categories

\begin{tabular}{|c|c|c|c|}
\hline & Higher complexity & Average (or uncertain) complexity & Lower complexity \\
\hline \multicolumn{4}{|l|}{ History } \\
\hline $\begin{array}{l}\text { S-Secondary therapies, surgery affecting } \\
\text { therapies-radiotherapy (RT) }\end{array}$ & $\begin{array}{l}\mathrm{RT} \text { in the past or current certain } \\
\text { indication }\end{array}$ & $\begin{array}{l}\text { Uncertain indication (pending final } \\
\text { post-operative pathology) }\end{array}$ & $\begin{array}{l}\text { No RT in the past or } \\
\text { non-indication }\end{array}$ \\
\hline $\begin{array}{l}\text { S-Surgery from the past including donor } \\
\text { sites }\end{array}$ & Malignant surgery & Benign ( \pm volume deficit $)$ & No past surgery \\
\hline $\begin{array}{l}\text { S-Sicknesses including diabetes, family } \\
\text { history }\end{array}$ & Co-morbidities & Family history only & $\begin{array}{l}\text { None/mild co- } \\
\text { morbidities }\end{array}$ \\
\hline S-Smoking & Current & Ex-smoker $>3$ months & Non-smoker \\
\hline S-Stay in hospital and recovery & Longer & Average \pm overnight & Shorter/day-case \\
\hline S-Social including work, leisure activities & Heavy & Sedentary & Regular \\
\hline $\begin{array}{l}\text { S-pSychological status and (s)Expectations } \\
\text { of the patient }\end{array}$ & Low and unrealistic & $\begin{array}{c}\text { Average/uncertain (may need } \\
\text { psychological assessment) }\end{array}$ & $\begin{array}{l}\text { High status and low } \\
\text { expectations }\end{array}$ \\
\hline \multicolumn{4}{|l|}{ Examination } \\
\hline $\begin{array}{l}\text { S-Shoulder limitation, Spine or chest-wall } \\
\text { deformity }\end{array}$ & Severe & Moderate & None \\
\hline $\begin{array}{l}\text { S-Scars and size (volume) of past tissue } \\
\text { removed }\end{array}$ & Multiple scars, significant defect & Some scars, minimal volume loss & None \\
\hline $\begin{array}{l}\text { S-Skin quality (elasticity, RT, dermal } \\
\text { thickness) }\end{array}$ & Poor & Moderate & Good \\
\hline S-Size (of tumour, breast, patient/BMI) & $\begin{array}{l}>4 \mathrm{~cm} \text { (pre or post-neo-adjuvant che- } \\
\text { motherapy), A-B cup bra }\end{array}$ & $2-4 \mathrm{~cm}, \mathrm{C}-\mathrm{D}$ cup bra & $\begin{array}{l}0-2 \mathrm{~cm}, \mathrm{E} \text { and larger } \\
\text { cup bra }\end{array}$ \\
\hline S-Site/s of tumour & Medial half or inner quadrants & Central & Lateral half or outer \\
\hline S-Sagging (ptosis) & Grade $3+$ & Grade 2 & Grade 1 \\
\hline $\begin{array}{l}\text { S-aSymmetry (including existing } \\
\text { a/symmetry) }\end{array}$ & $>2 \mathrm{~cm}$ off meridian & $1-2 \mathrm{~cm}$ & $0-2 \mathrm{~cm}$ \\
\hline
\end{tabular}

documenting and auditing contrasting pre-operative patient (subjective) expectations with realistic surgical (objective) possible goals and subsequently comparing the same with actual post-operative outcomes.

Table 2 Goals of surgery (expected or difficult or impossible)

\begin{tabular}{lll}
\hline $\begin{array}{l}\text { Goals of oncoplastic and } \\
\text { reconstructive surgery }\end{array}$ & $\begin{array}{l}\text { Pre-operative } \\
\text { Expected goals } \\
\text { (subjective/ } \\
\text { objective) }\end{array}$ & $\begin{array}{l}\text { Post-operative } \\
\text { Achievement of goals } \\
\text { (subjective/objective) }\end{array}$ \\
\hline
\end{tabular}

S-Size - foot-print espe-

cially base-width

S-Symmetry

S-Shape including of Nipple-areola complex

S-Sticking in front (Nipple position and symmetry)

S-Sensitivity of nipple and colour

S-Suckling (lactational)

function

S-Scar minimisation

For example, anticipated symmetry may not be possible in unilateral surgery but scar minimisation may be possible
Table 3 discusses common and the simplest decisionmaking as well as peri-operative processes for each complexity group of Table 1 in order to plan and achieve, realistically and safely, the goals in Table 2.

\section{History}

S-Secondary therapies, surgery-affecting therapies-Site
(radiotherapy), Systemic (chemotherapy or hormone therapy)

There is correlation between ORBS and oncological therapies (local and systemic). Increasingly, neo-adjuvant chemotherapy is initial therapy in younger patients, with tumours that are large, high-grade, estrogen receptornegative (with/without positive HER-2 receptor). Besides comparable survival benefit to adjuvant chemotherapy, neo-adjuvant chemotherapy can downsize tumour and facilitate BCS (breast-conserving surgery) when either mastectomy is a certainty or BCS indication is borderline. Good response may facilitate less extensive oncoplastic options in an existing BCS plan. Further, this approach precludes any delay in systemic therapy due to surgical complications. 
Table 3 Flowchart to support decision-making process based on individual complexity in order to achieve realistic goals and safe outcomes

Concepts and processes for optimising safe outcome

\begin{tabular}{|c|c|c|}
\hline $\begin{array}{l}\downarrow \\
\text { Higher complexity } \\
\text { (non-modifiable risk factors) }\end{array}$ & $\begin{array}{l}\downarrow \\
\text { Average complexity } \\
\text { (modifiable risk factors) }\end{array}$ & $\begin{array}{l}\downarrow \\
\text { Lower complexity } \\
\text { (avoidable risk factors) }\end{array}$ \\
\hline $\begin{array}{l}\text { Simpler breast-conserving techniques in the } \\
\text { presence of other risk factors such as } \\
\text { smoking (versus mammaplasty or perforator } \\
\text { flap partial reconstruction) }\end{array}$ & $\begin{array}{l}\text { Discuss pros and cons with patient—-shared } \\
\text { decision making } \\
\text { Allow reasonable time for patient to arrive at } \\
\text { a choice }\end{array}$ & $\begin{array}{l}\text { Dependent on anatomy and } \\
\text { pathology, all possible options } \\
\text { to be offered (whether available } \\
\text { locally or not) }\end{array}$ \\
\hline $\begin{array}{l}\text { Avoid symmetrising procedure } \\
\text { Avoid implant or free flap reconstructions } \\
\text { if anatomically all choices possible }\end{array}$ & $\begin{array}{l}\text { Minimise patient risk factors-(for example, } \\
\text { emphasise risk of re-starting smoking to a } \\
\text { recent ex-smoker during current anxiety phase) }\end{array}$ & $\begin{array}{l}\text { Maintain low risk by maximally } \\
\text { optimising peri-operative } \\
\text { techniques and processes }\end{array}$ \\
\hline $\begin{array}{l}\text { Consider delayed breast correction for } \\
\text { large breast-conserving surgery such as } \\
\text { fat grafting or partial reconstruction } \\
\text { Consider delayed breast reconstruction }\end{array}$ & $\begin{array}{l}\text { Minimise peri-operative risks such as pain, } \\
\text { immobility, length of stay }\end{array}$ & \\
\hline
\end{tabular}

Following BCS, adjuvant radiotherapy can cause breast parenchymal fibrosis, volume loss with resultant asymmetry and skin changes [2] with worse outcomes following surgical complications such as haematoma and seroma [3].

Post-mastectomy radiotherapy following implant-based IBR (immediate breast reconstruction) has up to four times higher complications [4]. However, the severity of capsular contracture rate is reportedly less with acellular dermal matrix (ADM) [5] reflecting some animal studies [6, 7]. Chemotherapy independently can adversely affect the type 1 collagen thereby affecting the remodelling and integration of ADM [8].

Although autologous delayed breast reconstruction (DBR) is an option where post-mastectomy radiotherapy is anticipated, increasingly deep inferior epigastric perforator (DIEP) flap is being recognised to withstand radiotherapy better over latissimus dorsi (LD) flap [9].

Patients with positive axillary nodes currently require axillary node dissection and are at higher odds of requiring adjuvant therapies. Separate axillary incision minimises seroma and infection of breast. Node negative patients may benefit from sentinel node biopsy prior to IBR.

\section{$S$-Surgery from the past including donor sites}

Elderly patients may not recall benign surgery from distant past, and therefore, leading questions can be informative. Previous breast incisions, volume loss, radiotherapy and duration since surgery may affect available oncoplastic options.

Abdominal scars may preclude pedicled (TRAM) or free (DIEP) flap reconstruction, but ingenious applications are possible [10]. CT angiogram is current gold standard [11, 12] for assessment of abdominal perforator vessels, though MR angiogram may provide better accuracy (without radiation exposure) [13]. Abdomen as a donor site in premenopausal patients should not preclude or compromise future pregnancies [14] but should be considered cautiously.

\section{S-Sicknesses_-personal and family history}

Co-morbidities such as diabetes and medications such as steroids [15] can cause wound healing issues especially in procedures involving multiple scars, e.g., therapeutic mammaplasty (Figs. 1 and 2) or flap-based breast reconstructions.

Patients with gene mutations or with high-risk family history may consider contra-lateral risk-reducing mastectomy, which in conjunction with oncological surgery may either increase or decrease reconstructive options.

\section{S-Smoking}

Smoking, independently, is a risk factor [15-17] for wound healing besides systemic peri-operative morbidities. Immediate post-operative risks of partial/total nipple/skin necrosis are higher than the non-smoking population $[15,16]$.

Wound complications may delay adjuvant radiotherapy/ chemotherapy; hence, oncological surgery alone may be the safer initial option followed by DBR or symmetrisation [16]. Provision of counselling and support for smoking cessation is therefore imperative.

\section{S-Stay in hospital and recovery}

Many patients prefer shorter surgery with consequent shorter hospital stay and faster recovery. They may not prefer any aesthetically corrective BCS (mammaplasty, etc.) or reconstructive procedure and choose to have oncological 


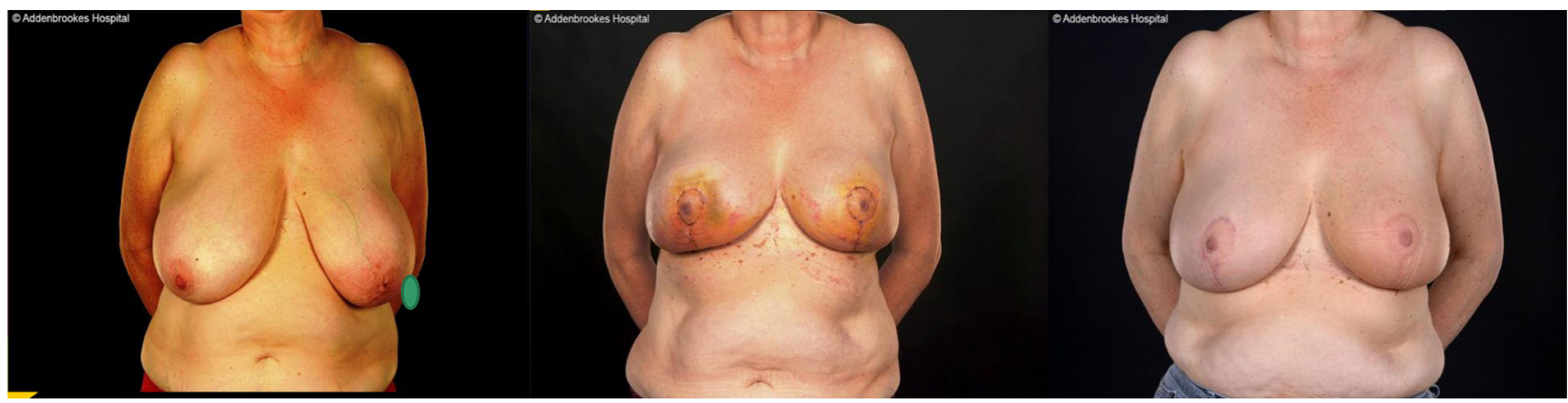

Fig. 1 Left wide local excision and therapeutic mammaplasty and right symmetrising reduction (wise-pattern). Left therapeutic mammaplasty (wise-pattern based on extended supero-medial pedicle) for lower outer quadrant multifocal tumours ( 32 and $12 \mathrm{~mm}$ being $23 \mathrm{~mm}$ apart) following neo-adjuvant chemotherapy. The excision weight was $523 \mathrm{~g}$ and the right symmetrising reduction weight was $616 \mathrm{~g}$. Both breasts were E cups with grade 3 ptosis pre-operatively with acceptable post-operative symmetry in and out of bra. The bottom panel shows post-operative pictures at 2 weeks (bottom left) post-operatively and at 4 months (bottom right) following radiotherapy extirpation surgery guaranteeing them shorter hospital stay. For similar reasons, they may prefer implant to other complex reconstructions.

\section{S-Social including work, leisure activities}

Certain surgical options may not be preferred or suitable dependent on patient's family/relationship commitments or business/leisure activities. Active patients even with lower tumour-to-breast ratio may prefer therapeutic mammaplasty with symmetrising reduction.

Similarly, autologous reconstruction (LD, TRAM or TUG/gluteal flaps) may not be suitable in patients with heavy physical occupations or leisure activities (e.g., rock-climbing, tennis) [18] particularly LD flap on dominant hand-side. Equally, concerns regarding possible implant movement or hyper-animation may influence patient decision. Newer tabbed prosthesis [19] or prepectoral implants with complete acellular dermal matrix (ADM) cover [20] may minimise such movements, but larger series are necessary.

\section{S-pSychological status and (s)expectations of the patient}

Basic assessment and understanding of patient's psychological status concerning comprehension, retention and discrimination between surgical options are essential after provision of all written information and sufficient time. The shock of
Fig. 2 Left wide local excision and therapeutic mammaplasty and right symmetrising reduction (batwing pattern). Left wide local excision and therapeutic mammaplasty (for a pre-operative $3-\mathrm{cm}$ tumour which was $6 \mathrm{~cm}$ postoperatively but with clear margins) and right reduction performed in bat-wing mastopexy fashion due to D cup breasts, gross ptosis, high set infra-mammary folds, long sternalnipple distance and thin skin along with high body mass index. A skinonly peri-areolar mastopexy and skin-only mastopexy medial to nipple-areola complex with total excision weight of $149 \mathrm{~g}$ on the therapeutic mammaplasty side allowed acceptable results (3 weeks post-operatively) in a difficult case

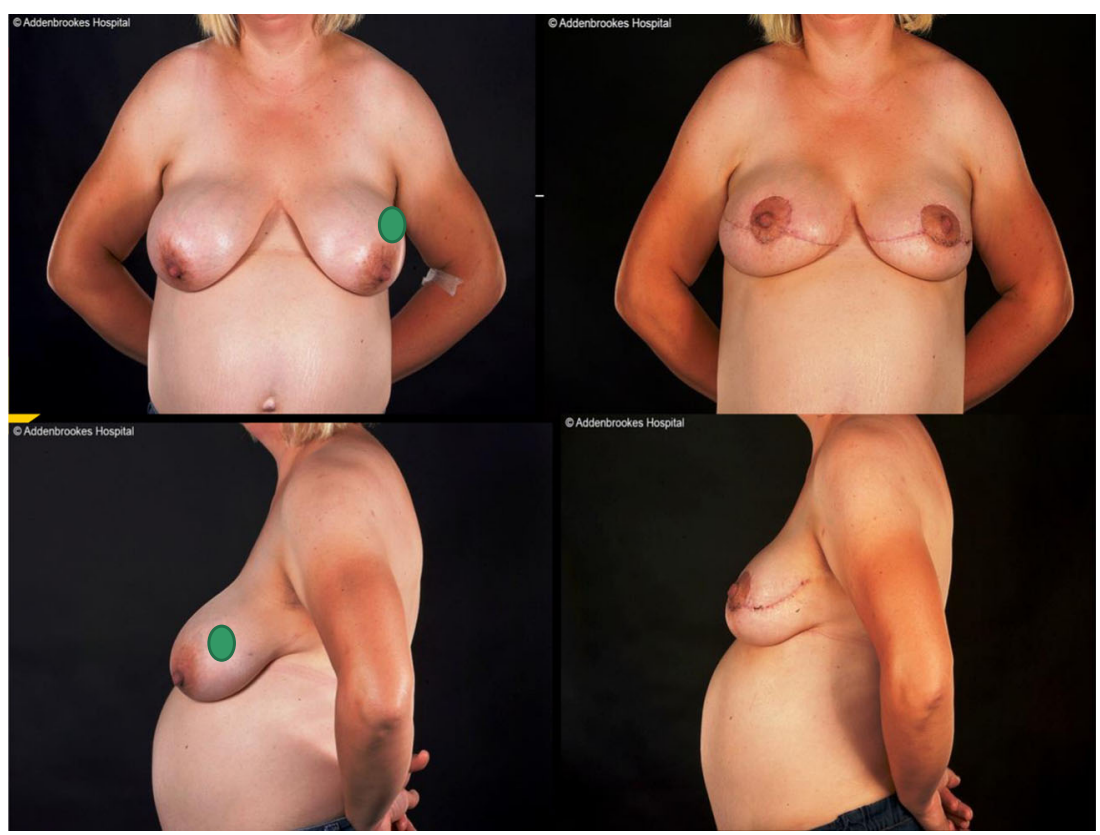


cancer diagnosis may preclude reasoned decision initially. If deemed necessary, formal psychological assessment may be necessary [21] especially prior to risk-reducing surgery. This may be particularly necessary in non-high-risk family history patients who increasingly request contra-lateral prophylactic mastectomy where decision-making process may be in question [22]. When patient expectations do not meet oncological or surgical safety, it is appropriate to re-discuss options in a subsequent multi-disciplinary meeting (MDT). Breast care nurses during their one-to-one consultation can be better in ascertaining patient's expectations in terms of body image, sexuality and relationship factors influencing patient choice $[23,24]$.

However, if there is an ambiguity or unavailability of an oncoplastic surgical option locally, UK national oncoplastic guidelines [1] recommend that patient is referred to another centre. The patient, however, loses if the initial surgeon is unable to recognise oncoplastic options due to current unavailability of an organised comprehensive approach to assessment.

\section{Examination}

\section{S-Shoulder limitation or spine or chest wall deformity}

Pre-existing spinal deformity or shoulder movement limitation may predispose to possible post-operative morbidity (following LD/abdominal flap). In addition, any existing spinal asymmetry may cause false impression of breast asymmetry. Therefore, during measurements and marking, both clavicles and both shoulder tips should be at the same horizontal plane. Usual involuntary tendency for the ipsilateral shoulder to be withdrawn superiorly during breast palpation (mainly due to post-biopsy tenderness/bruising) could be minimised if explained to patients.

The newer lateral chest wall perforator (LCWP) flaps [25] (Figs. 3 and 4) which utilise the oft-redundant lateral chest wall subcutaneous (non-muscular) tissue flap based on perforators increase the options in small breasts with pre-existing musculo-skeletal limitations. These (essentially replacing mini-LD flaps) can be used for either whole breast or commonly partial (Figs. 3 and 4) breast reconstructions [26] in high tumour-to-breast ratio BCS.

\section{S-Scars if any and size (volume) of previous tissue removed}

Previous scar/s may preclude certain oncoplastic BCS or mastectomy reconstruction. For example, previous peri-areolar incision (from therapeutic or cosmetic reductions) including superior half of the circumference may preclude supero-medial/ superior pedicle mammaplasty/reduction [27].

Similarly, pre-existing scars during skin/nipple sparing mastectomy may be an additional risk to skin flap vascularity particularly in direct-to-implant (Fig. 5) IBR [27, 28]. In contrast, an initial reduction/mastopexy (with associated scars) in large ptotic breasts can achieve an optimal skin envelope before mastectomy reconstruction (particularly in risk-reducing setting) though both components are achievable in a single stage [29, 30].

Augmented (implants) breasts with new cancer will have minimal scars, but achieving volume symmetry in BCS or planning BCS or mastectomy can be challenging [31]. Explantation with or without mastopexy may be necessary to avoid capsular contracture following adjuvant RT in invasive disease. However, iatrogenic damage to existing implant or rarely tumour infiltration may result in unplanned explantation. As discussed earlier in the history section, donor site scars are equally important when planning flaps for partial or total breast reconstruction.

\section{S-Skin quality (stretch-elasticity, sunburn-radiotherapy, dermal thickness)}

Striae over the breasts following pregnancy or weight loss render the skin thin and ptotic. In some patients, this may necessitate skin-only mastopexy, either wholly or partly (Fig. 2) following BCS (to provide a better shape) with or without contralateral symmetrisation. In contrast, however, skin with limited thickness of the dermis limits oncoplastic options and may contribute to wound complications [16] including following reconstructions (especially implant-based reconstructions). Similarly, striae on large skin island (necessitated in DBR) abdominal wall flap compromises overall aesthetic outcome.

Irradiated skin (mantle radiotherapy for lymphoma or previous BCS or following mastectomy) may have limited elasticity and unpredictable vascularity particularly from recent radiotherapy. There is higher risk of wound dehiscence especially at the $\mathrm{T}$-junction with wise-pattern reduction mammaplasty. DBR in irradiated breast has traditionally been two-stage implant-based reconstruction, but there are increasingly large series of ADM-based single-stage implant reconstructions with either permanent or expandable implant $[15$, $32,33]$.

\section{$S$-Size of breast and patient}

The breast size vis-à-vis tumour size, fluctuating body weight along with existing asymmetry in size, shape and nipple position, influences outcome though patients may be unaware of subtle asymmetry particularly breast shape and nipple position.

In general, lower tumour-to-breast ratio allows "displacement" (Figs. 1 and 2) type of oncoplastic options following BCS, whilst higher ratio necessitates "replacement", either immediate (Fig. 3) or delayed (Fig. 4) partial [34] or total reconstruction (Fig. 5). Concurrent or delayed augmentation of small breasts or reduction of large breasts may achieve or maintain symmetry (Fig. 5). 
Fig. 3 Immediate partial reconstruction of left breast with lateral intercostal artery perforator (LiCAP) flap. Wide local excision of $19 \mathrm{~mm}$. Grade 3 tumour in the axillary tail area of a 36 year-old woman would have left a defect if uncorrected especially following boost radiotherapy. However, corrected and overcompensated with LiCAP flap to correct hollowness in axillary tail area (as pointed on the right side) avoids deformity from alternative of parenchymal mobilisation as the nipple-areola complex would have been displaced in an otherwise good shape breasts with no ptosis. The 3 weeks post-operative picture shows blue discolouration from patent blue dye for sentinel lymph node biopsy (which was negative)

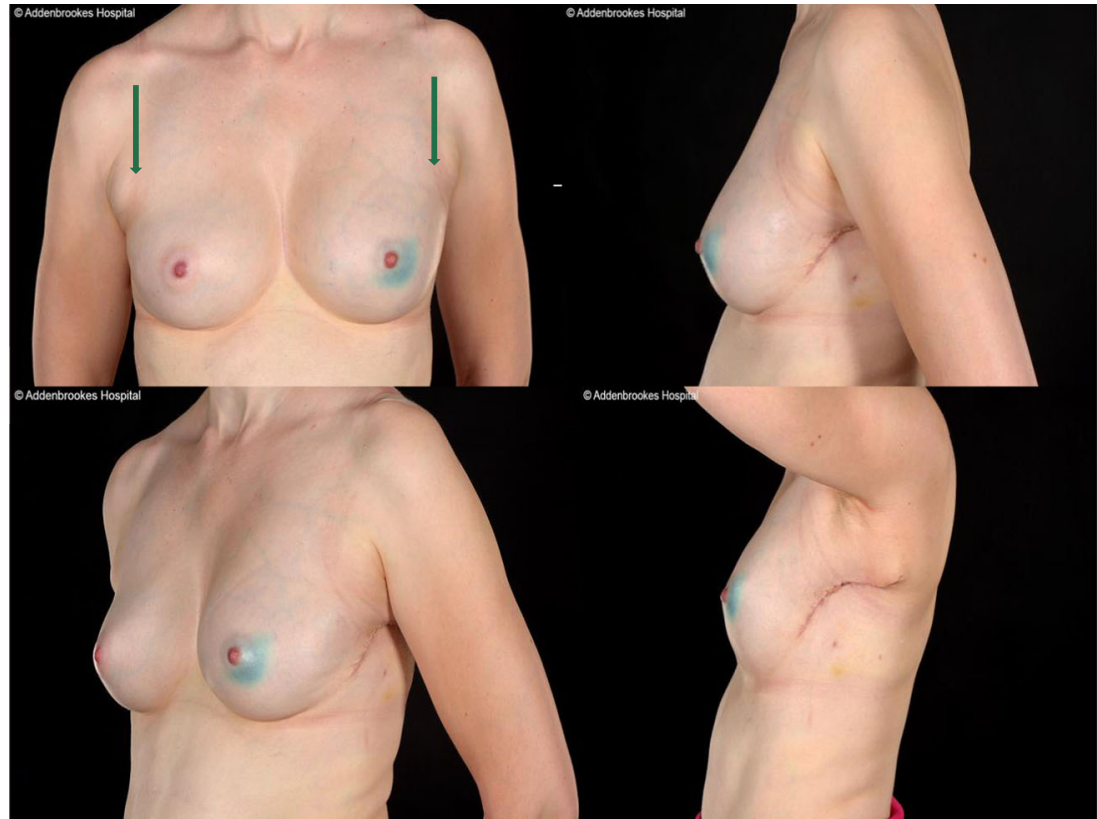

In general, though not strictly, large wide-base breasts are more suitable for wise-pattern mammaplasty (Fig. 1) whilst medium narrow-base breasts for vertical-scar mammaplasty. However, other patterns such as bat-wing (Fig. 2) mammaplasty may be useful. In small to moderate size breasts with higher tumour-to-breast ratio, a variety of volume replacement flaps are available and increasingly conservative (from muscular to perforator flaps) [35].
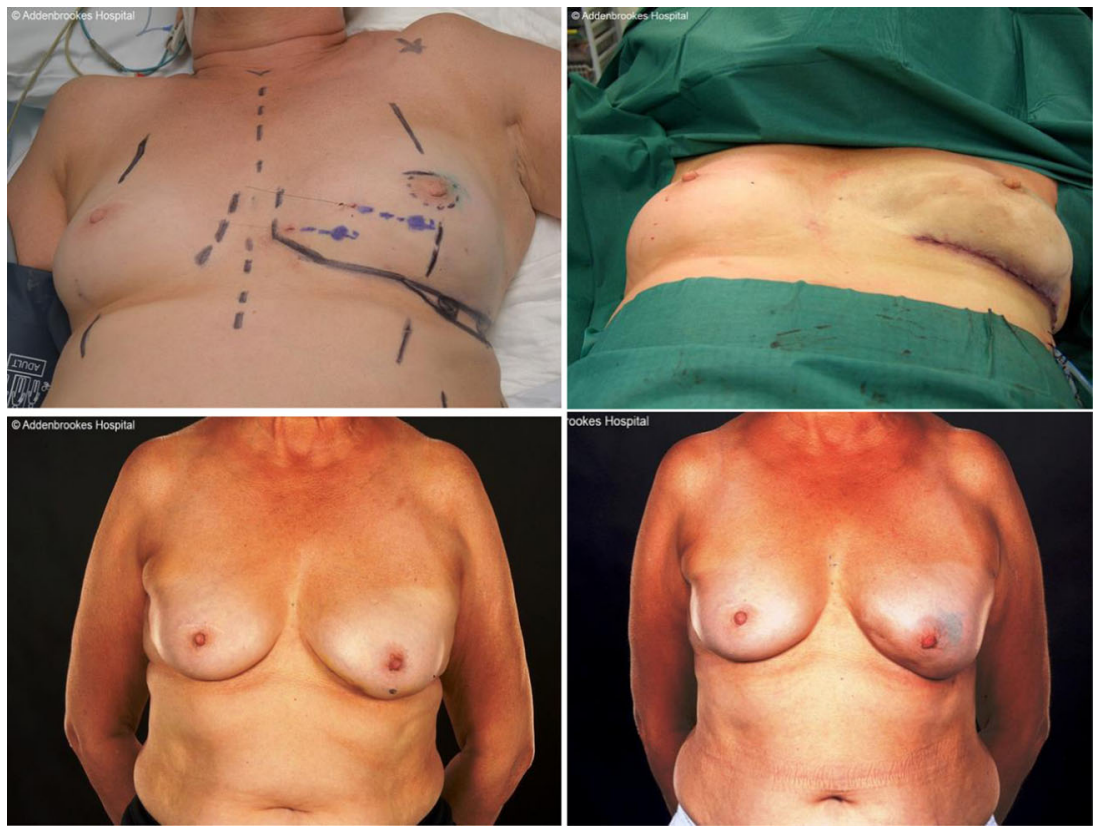

Fig. 4 Delayed immediate partial reconstruction of left breast with lateral thoracic artery perforator (L-TAP) flap. Wide local excision (specimen weight was $82 \mathrm{~g}$ ) for lower inner quadrant multi-focal (grade $1,8 \mathrm{~mm}$; and grade 2,14 $\mathrm{mm}$ being $28 \mathrm{~mm}$ apart) tumours (shown by two blue dots with localising wires in situ) was performed initially. Following confirmation of clear margins, 4 weeks after initial surgery, a second-stage L-TAP flap partial breast reconstruction was performed. Excision alone would have caused unacceptable deformity, whilst other patterns such as

radial with re-centralisation of nipple may have even corrected existing symmetry, but the infra-mammary scar did not disturb the existing footprint of the breast, increase the risks or obviate any reconstruction, if mastectomy was necessitated (higher than average chances of positive margins due to multi-focal tumours). The bottom panel on the left is preoperative, while the right is post-operative picture. The 6 weeks postoperative picture shows blue discolouration (bottom right) from patent blue for sentinel lymph node biopsy (which was negative) 


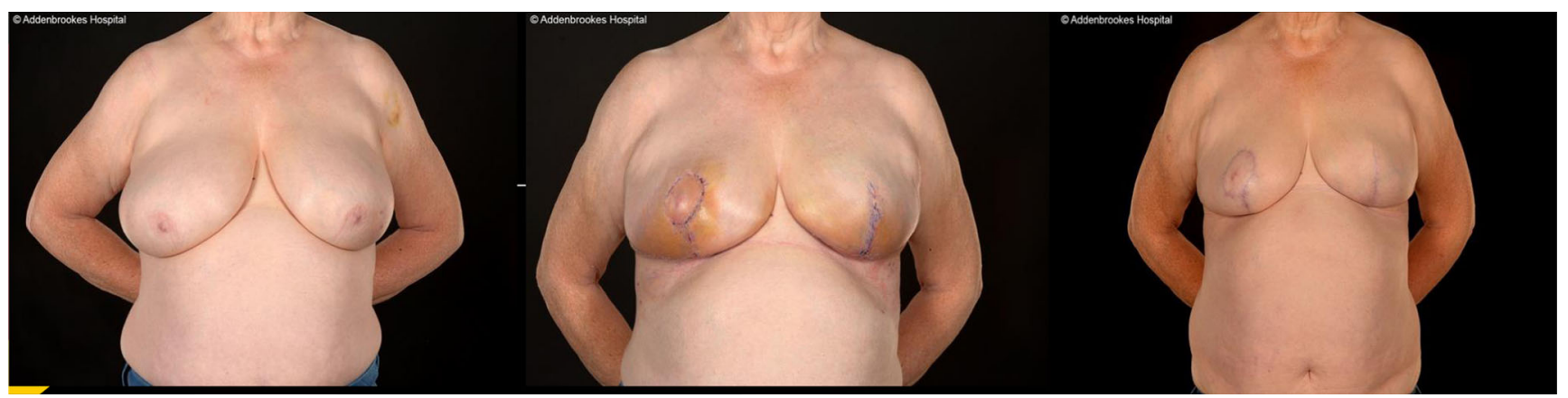

Fig. 5 Left skin-sparing wise-pattern mastectomy with inferior pole dermal sling based direct to (permanent) implant reconstruction with symmetrising reduction. Left skin-sparing mastectomy and inferior dermal-based sling using a permanent anatomical implant (Allergan ${ }^{\mathrm{TM}}$
$320 \mathrm{MM})$ and symmetrising wise-pattern reduction. Post-operative pictures are top right (4 weeks), bottom left (4 weeks) and bottom right (6 months)
In medium size breasts, post-mastectomy autologous reconstructions with or without an implant are options. However, in large ptotic breasts with inadequate autologous tissue, lower pole dermal sling based implant reconstruction is one option including direct-to-implant [36] (Fig. 5). In large breasts, a recent modification of dermal-based reconstruction ("Goldilocks" mastectomy) involves utilising de-epithelised dermis without prosthesis to reconstruct a breast mound [37].

High BMI in addition to increased risk of peri-operative morbidities is an independent risk factor for both reduction/ mammaplasty [16] and implant-based reconstructions (both ADM and non-ADM) [15, 38]. Further, weight loss may render the breast more ptotic with thinner skin (generally with striae) with its potential implications as discussed in the preceding section [16].

\section{S-Site of tumour in the breast}

Upper outer quadrant is the commonest site for breast cancer and generally the simplest to manage with either curvilinear or a radial incision with or without nipple-areolar complex recentralisation. Lateral fold approach is an option provided there is no compromise in oncological resection. Further upper/lower outer tumours, if likely large enough to leave a defect or cosmetic deformity, may necessitate LCWP flap (Fig. 3).

Upper inner quadrant tumours are challenging and options include incision at peri-areola or curvilinear incision as away from cleavage as possible. For lower half of the breast, peri-areolar, lateral/infra-mammary fold or lower vertical scars are options. However, loss of volume can lead to bird's beak deformity that may be preventable by local tissue re-arrangement, mammaplasty or LCWP flap (Fig. 4). Mammaplasty pedicle options are quite dependent on tumour location and matter of extensive discussion. Central tumours often necessitate excision of the nipple-areola complex with options ranging from simple parenchymal mobilisation through mammaplasty to replacement flaps [39].

Tumour site acquires relevance in skin sparing (incision vis-à-vis skin involvement, if any) especially in nipplesparing (or subcutaneous) mastectomy [17].

\section{S-Sagging (ptosis)}

Large ptotic breasts have higher risk of NAC necrosis following reduction mammaplasty [16]. Those with sternal notch to nipple distance of $>26 \mathrm{~cm}$ have higher incidence of skin flap necrosis following skin sparing mastectomy [40], and alternative techniques such as skin-only mastopexy may be necessary for a safe outcome (Fig. 2).

Small to moderate breasts with minimal ptosis are generally suitable for implant-based (with or without ADM) reconstruction whilst large ptotic breasts for either BCS (mammaplasty) or dermal sling-based implant reconstructions (Fig. 5) [36] as discussed previously. However, ptosis due to predominant volume loss (post-partum or weight loss), resulting in redundant skin, may necessitate either mastopexy only BCS (Fig. 2) or two-staged mastopexy implant-based reconstruction [29, 30]. It is difficult to match natural ptosis with any reconstruction especially implant-based reconstruction.

\section{S-aSymmetry}

Besides often obvious asymmetry in size, appraisal of differences in breast shape and nipple position helps planning minimisation of post-operative asymmetry. It is important to discuss these subtle asymmetries with the patient for contrasting expectations versus realistic possible outcomes.

If the affected size is larger, size asymmetry in reduction mammaplasty can provide better post-operative symmetry. 
However, if the affected side is smaller, then either contralateral reduction or an ipsilateral replacement flap may be necessary.

Gross shape asymmetry (e.g., tuberous deformity) may necessitate extensive surgery including implant-based reconstructions with or without lipofilling. Lipofilling has been shown to be technically safe in some large series [41], and the usage in cancer extirpative surgery is increasing [42].

Asymmetry of nipple position can be subtle, and even if symmetrical, nipples may not be aesthetically positioned and could be either medial or lateral to the breast meridian. It may be possible to correct para-median nipple positions during mammaplasty/reduction, but inherent above-average nipple mobilisation adds additional risk to nipple vascularity.

\section{Goals of surgery}

\section{S-Size (base side-ways/width and stand/height measurement)}

Post-operative breast size should be aesthetically proportional to patient's frame/torso. The goals of cosmetic surgery might not be possible or equivalent to goals in cancer surgery. For example, (cosmetic) augmentation disproportionately larger to a woman's petite frame may be possible, but there will be limitations in achieving a similar breast size following reconstruction.

Most surgeons regard larger and wider breasts suitable for wise-pattern mammaplasty whilst narrower and smaller breasts suitable for vertical-scar mammaplasty. However, the aim of therapeutic mammaplasty is to achieve reduction of skin and parenchyma at the same time as BCS. Therefore, instead of a default template (Figs. 1 and 2), mammaplasty should be tailored according to the individual breast morphology as discussed already (skin thickness, elasticity, ptosis).

Similarly, during implant-based reconstruction, the size or the volume of prosthesis is dependent on firstly width and secondly height of the breast footprint. In autologous reconstruction, the pliability of the autologous tissue may allow moulding and volume adjustment within the undisturbed (in immediate) or recreated (in delayed) breast footprint and envelope.

\section{S-Shape}

The foremost goal of any ORBS procedure is a good shape. For a good aesthetic outcome, good shape of an individual breast is more important than the size. However, for bilateral procedures, achieving both shape and size symmetry are important though challenging (Fig. 5) especially when the surgery is unilateral whether BCS or reconstruction (particularly an implantbased reconstruction which even if symmetrical initially will lag behind the progressive ptosis with time in the contra-lateral breast). Therefore, it is important to point out to the patients that good symmetry achieved in the short-term may not persist longterm. This is nigh-certain with BCS followed by radiotherapy and mastectomy with implant-based reconstruction. Explanation of this ptosis phenomenon will allow patients make an informed choice regarding surgery to the unaffected breast.

The third dimension of projection is usually dependent on the permutation and combination of the initial two measurements in both mammaplasty and reconstructions (especially in implant). Estimated projection in implant reconstruction is an important variable for symmetry, if unilateral. Magnitude of projection is relevant in a small breast with thin skin to avoid unnecessary skin stretch and compromise vascularity.

\section{S-Symmetry}

Following BCS, medium- and long-term radiotherapy effects lead to firmer, smaller and less ptotic breasts versus the contralateral unaffected breast. As discussed above, this usually leads to long-term effects on shape asymmetry and may or may not lead to global volume asymmetry. If resultant volume loss causes asymmetry, contra-lateral reduction is an option at a later stage though increasingly the modern practice is towards concurrent symmetrisation (Figs. 1 and 2). Similarly, a concurrent symmetrising procedure (reduction or augmentation) during IBR is an option (Fig. 5). As discussed above (in the shape section), many patients choose concurrent symmetrisation to avoid another surgery, hospital admission, and a particular wish to have a sense of closure in terms of treatment of breast cancer and associated therapies.

\section{S-Sticking in front = nipple position and symmetry}

It is important to plan a shape that allows for optimal position of nipple on the mound. Amy mammaplasty technique will incorporate nipple positioning during marking/planning. However, planning such in reconstruction is more difficult. Sub-optimal ratio between upper and lower pole of breast mound may occur due to displacement of scar (in skinsparing mastectomy) or nipple (in nipple-sparing mastectomy) especially when reconstruction includes expansion process.

\section{S-Sensitivity of nipple}

Partial loss of nipple sensation is inevitable in mammaplasty or nipple-sparing mastectomy, whilst total loss of nipple sensation is common. Many patients report restoration of sensation that initially could be abnormal and para-aesthetic. Septum-based reductions can preserve nipple sensation [43], but application of such technique for mammaplasty following $\mathrm{BCS}$ in cancer may not be possible.

\section{S-Suckling (lactational) function}

A small prospective study found lowered breast feeding rates following reduction (and augmentation) though possibly related to patient profile and acceptance [44]. However, a systematic 
review of the effect of reduction on breast-feeding in the first post-partum month did not show any difference compared to non-reduction population [45] though there may be subtle differences between pedicles [46]. However, data is unavailable in therapeutic mammaplasty in cancer setting.

Regardless, oncological principles especially with tumours close to nipple-areola complex take priority in BCS over any planned reduction mammaplasty. Therefore, in young women who have not started or completed family, and wish to preserve breast-feeding function, simple BCS may be the only option. Some data suggests that radiotherapy can reduce lactation in less than $50 \%$ patients including lactational volume [47]. Therefore, extensive surgery such as mammaplasty may reduce lactational ability further especially when combined with contra-lateral symmetrising reduction. It is imperative that the patient makes informed choice between aesthetic vis-à-vis functional outcomes.

\section{S-Scar minimisation}

Factors such as shape and size are important to surgeons, but occasionally, dominating concern of the patient remains the extent of the scar. Dependent on the type, there will be limited options for scar pattern in mastectomy. In BCS, scar pattern depends on tumour location, breast size, shape, ptosis, etc. (as already discussed). However, if the goal of parenchymal reduction is achievable with any scar pattern, then utilising a pattern with minimal scar becomes prudent.

\section{Conclusions}

This review discusses the pros and cons of a wide range of scenarios with possible ORBS options in an organised manner. Although not exhaustive, this organised approach may allow rapid yet comprehensive oncoplastic assessment minimising inadvertent exclusion of any common variable. As a decision-making tool, it should enable senior trainees/ inexperienced consultants establish baseline facts and differentiate between options helping arrive at a shared-decision with the patient. It could also be utilised as an audit tool to ensure quality of oncoplastic assessment whilst ensuring availability of all possible options to all patients in any centre.

Further to this article, the aim is to validate this chart retrospectively in a large cohort of post-operative patients managed by different surgeons followed by prospective validation study.

\section{Compliance with ethical standard}

Conflict of interest The author Amit Agrawal declares that there is no conflict of interest in the preparation of this manuscript.

Ethical standards For this type of article formal consent from a local ethics committee is not required.
Patient consent Patients provided written consent for the use of their images.

Funding source None.

Open Access This article is distributed under the terms of the Creative Commons Attribution 4.0 International License (http:// creativecommons.org/licenses/by/4.0/), which permits unrestricted use, distribution, and reproduction in any medium, provided you give appropriate credit to the original author(s) and the source, provide a link to the Creative Commons license, and indicate if changes were made.

\section{References}

1. Rainsbury D, Willett A (eds) (2012) Oncoplastic breast reconstruction: guidelines for best practice. ABS \& BAPRAS, UK. http://www.associationofbreastsurgery.org.uk/media/23851/final oncoplastic_guidelines_for_use.pdf

2. Mukesh M, Harris E, Jena R, Evans P, Coles C (2012) Relationship between irradiated breast volume and late normal tissue complications: a systematic review. Radiother Oncol 104(1):1-10

3. Mukesh MB, Barnett G, Cumming J et al (2012) Association of breast tumour bed seroma with post-operative complications and late normal tissue toxicity: results from the Cambridge Breast IMRT trial. Eur J Surg Oncol 38(10):918-924

4. Salzberg CA, Ashikari AY, Koch RM, Chabner-Thompson E (2011) An 8-year experience of direct-to-implant immediate breast reconstruction using human acellular dermal matrix (AlloDerm). Plast Reconstr Surg 127(2):514-524

5. Israeli R, Feingold RS (2011) Acellular dermal matrix in breast reconstruction in the setting of radiotherapy. Aesthet Surg J 31(7 Suppl):51S-64S

6. Schmitz M, Bertram M, Kneser U, Keller AK, Horch RE (2013) Experimental total wrapping of breast implants with acellular dermal matrix: a preventive tool against capsular contracture in breast surgery? J Plast Reconstr Aesthet Surg 66(10):1382-1389

7. Stump A, Holton LH 3rd, Connor J, Harper JR, Slezak S, Silverman RP (2009) The use of acellular dermal matrix to prevent capsule formation around implants in a primate model. Plast Reconstr Surg 124(1):82-91

8. Myckatyn TM, Cavallo JA, Sharma K et al (2015) The impact of chemotherapy and radiation therapy on the remodeling of acellular dermal matrices in staged, prosthetic breast reconstruction. Plast Reconstr Surg 135(1):43e-57e

9. Taghizadeh R, Moustaki M, Harris S, Roblin P, Farhadi J (2015) Does post-mastectomy radiotherapy affect the outcome and prevalence of complications in immediate DIEP breast reconstruction? A prospective cohort study. J Plast Reconstr Aesthet Surg 68(10): $1379-85$

10. Hamdi M, Larsen M, Craggs B, Vanmierlo B, Zeltzer A (2014) Harvesting free abdominal perforator flaps in the presence of previous upper abdominal scars. J Plast Reconstr Aesthet Surg 67(2): 219-225

11. Teunis T, van Voss MR H, Kon M, van Maurik JF (2013) CTangiography prior to DIEP flap breast reconstruction: a systematic review and meta-analysis. Microsurgery 33(6):496-502

12. Keys KA, Louie O, Said HK, Neligan PC, Mathes DW (2013) Clinical utility of CT angiography in DIEP breast reconstruction. J Plast Reconstr Aesthet Surg 66(3):e61-65

13. Cina A, Barone-Adesi L, Rinaldi P et al (2013) Planning deep inferior epigastric perforator flaps for breast reconstruction: a 
comparison between multidetector computed tomography and magnetic resonance angiography. Eur Radiol 23(8):2333-2343

14. Patel KM, Basci D, Nahabedian MY (2013) Multiple pregnancies following deep inferior epigastric perforator (DIEP) flap breast reconstruction. J Plast Reconstr Aesthet Surg 66(3):434-436

15. Ibrahim AM, Shuster M, Koolen PG et al (2013) Analysis of the National Surgical Quality Improvement Program database in 19, 100 patients undergoing implant-based breast reconstruction: complication rates with acellular dermal matrix. Plast Reconstr Surg 132(5):1057-1066

16. Robert G, Duhamel A, Alet JM, Pelissier P, Pinsolle V (2014) Complications of breast reduction about 715 breasts. Ann Chir Plast Esthet 59(2):97-102

17. Agrawal A, Sibbering DM, Courtney CA (2013) Skin sparing mastectomy and immediate breast reconstruction: a review. Eur J Surg Oncol 39(4):320-328

18. Lutringer J, Flipo B, Carles M, Gal J, Chignon-Sicard B (2012) Functional impairment and its impact on sporting activities after latissimus dorsi flap breast reconstruction. Ann Chir Plast Esthet 57(6):567-574

19. Spear SL, Economides JM, Shuck J, Patel KM (2014) Analyzing implant movement with tabbed and nontabbed expanders through the process of two-stage breast reconstruction. Plast Reconstr Surg 133(3):256e-260e

20. Reitsamer R, Peintinger F (2015) Prepectoral implant placement and complete coverage with porcine acellular dermal matrix: a new technique for direct-to-implant breast reconstruction after nipple-sparing mastectomy. J Plast Reconstr Aesthet Surg 68(2): 162-167

21. NICE: Breast Cancer Quality Standard. https://www.nice.org. uk/guidance/QS12 2011.

22. Parker PA, Peterson SK, Bedrosian I et al: Prospective study of surgical decision-making processes for contralateral prophylactic mastectomy in women with breast cancer. Ann Surg 2015

23. Arving C, Sjoden PO, Bergh J et al (2007) Individual psychosocial support for breast cancer patients: a randomized study of nurse versus psychologist interventions and standard care. Cancer Nurs 30(3):E10-19

24. Remmers H, Holtgrawe M, Pinkert C (2010) Stress and nursing care needs of women with breast cancer during primary treatment: a qualitative study. Eur J Oncol Nurs 14(1):11-16

25. Thomsen JB, Gunnarsson GL (2014) The evolving breast reconstruction: from latissimus dorsi musculocutaneous flap to a propeller thoracodorsal fasciocutaneous flap. Gland Surg 3(3):151-154

26. Hamdi M, Van Landuyt K, Hijjawi JB, Roche N, Blondeel P, Monstrey S (2008) Surgical technique in pedicled thoracodorsal artery perforator flaps: a clinical experience with 99 patients. Plast Reconstr Surg 121(5):1632-1641

27. Vaughn CJ, Peled AW, Esserman LJ, Foster RD: Feasibility of performing total skin-sparing mastectomy in patients with prior circumareolar mastopexy or reduction mammoplasty incisions. Ann Plast Surg 2013

28. Alperovich M, Tanna N, Samra F et al (2013) Nipple-sparing mastectomy in patients with a history of reduction mammaplasty or mastopexy: how safe is it? Plast Reconstr Surg 131(5):962-967

29. Al-Mufarrej FM, Woods JE, Jacobson SR (2013) Simultaneous mastopexy in patients undergoing prophylactic nipple-sparing mastectomies and immediate reconstruction. J Plast Reconstr Aesthet Surg 66(6):747-755
30. Beale EW, Ramanadham S, Harrison B, Rasko Y, Armijo B, Rohrich RJ (2014) Achieving predictability in augmentation mastopexy. Plast Reconstr Surg 133(3):284e-292e

31. Tuli R, Flynn RA, Brill KL, Sabol JL, Usuki KY, Rosenberg AL (2006) Diagnosis, treatment, and management of breast cancer in previously augmented women. Breast J 12(4):343-348

32. Clemens MW, Kronowitz SJ (2012) Acellular dermal matrix in irradiated tissue expander/implant-based breast reconstruction: evidence-based review. Plast Reconstr Surg 130(5S-2):27S-34S

33. Kim JYS, Davila AA, Persing S et al (2012) A meta-analysis of human acellular dermis and submuscular tissue expander breast reconstruction. Plast Reconstr Surg 129(1):28-41

34. Clough K, Kaufman G, Nos C, Buccimazza I, Sarfati I (2010) Improving breast cancer surgery: a classification and quadrant per quadrant atlas for oncoplastic surgery. Ann Surg Oncol 17(5): $1375-1391$

35. Lee JW, Kim MC, Park HY, Yang JD (2014) Oncoplastic volume replacement techniques according to the excised volume and tumor location in small- to moderate-sized breasts. Gland Surg 3(1): 14-21

36. Nava MB, Ottolenghi J, Pennati A et al (2012) Skin/nipple sparing mastectomies and implant-based breast reconstruction in patients with large and ptotic breast: oncological and reconstructive results. Breast 21(3):267-271

37. Richardson $\mathrm{H}, \mathrm{Ma} \mathrm{G}$ (2012) The Goldilocks mastectomy. Int J Surg 10(9):522-526

38. Ho G, Nguyen TJ, Shahabi A, Hwang BH, Chan LS, Wong AK (2012) A systematic review and meta-analysis of complications associated with acellular dermal matrix-assisted breast reconstruction. Ann Plast Surg 68(4):346-356

39. Park HC, Kim HY, Kim MC et al (2014) Partial breast reconstruction using various oncoplastic techniques for centrally located breast cancer. Arch Plast Surg 41(5):520-528

40. Davies K, Allan L, Roblin P, Ross D, Farhadi J (2011) Factors affecting post-operative complications following skin sparing mastectomy with immediate breast reconstruction. Breast 20(1):21-25

41. Delay E, Garson S, Tousson G, Sinna R (2009) Fat injection to the breast: technique, results, and indications based on 880 procedures over 10 years. Aesthet Surg J 29(5):360-376

42. Petit JY, Rietjens M, Botteri E et al (2013) Evaluation of fat grafting safety in patients with intraepithelial neoplasia: a matched-cohort study. Ann Oncol 24(6):1479-1484

43. Hamdi M, Van Landuyt K, Tonnard P, Verpaele A, Monstrey S (2009) Septum-based mammaplasty: a surgical technique based on Wuringer's septum for breast reduction. Plast Reconstr Surg 123(2):443-454

44. Andrade RA, Coca KP, Abrao AC (2010) Breastfeeding pattern in the first month of life in women submitted to breast reduction and augmentation. J Pediatr (Rio J) 86(3):239-244

45. Thibaudeau S, Sinno H, Williams B (2010) The effects of breast reduction on successful breastfeeding: a systematic review. J Plast Reconstr Aesthet Surg 63(10):1688-1693

46. Kakagia D, Tripsiannis G, Tsoutsos D (2005) Breastfeeding after reduction mammaplasty: a comparison of 3 techniques. Ann Plast Surg 55(4):343-345

47. Leal SC, Stuart SR, Carvalho Hde A (2013) Breast irradiation and lactation: a review. Expert Rev Anticancer Ther 13(2):159-164 